International Journal of Pure and Applied Mathematics

Volume 105 No. 1 2015, 73-86

ISSN: 1311-8080 (printed version); ISSN: 1314-3395 (on-line version)

url: http://www.ijpam.eu

doi: http://dx.doi.org/10.12732/ijpam.v105i1.7

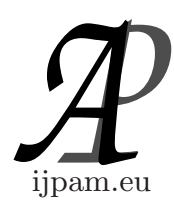

\title{
ADJACENCY IN THE LATTICE OF ČECH CLOSURE OPERATORS
}

\author{
M. Kunheenkutty ${ }^{1}$, T. Kavitha ${ }^{2}$, P.T. Ramachandran ${ }^{3}$ \\ ${ }^{1}$ Anappilakkal(H), Pullancheri, Kuzhimana P.O. \\ Malappuram Dt., Pin:673641, Kerala, INDIA \\ ${ }^{2,3}$ Department of Mathematics \\ University of Calicut \\ Malappuram Dt., Pin: 673635, Kerala, INDIA
}

\begin{abstract}
In this paper we investigate the adjacency relations in the lattice of $\check{C}$ ech closure operators on a fixed set with special reference to $T_{1} \check{C}$ ech closure operators. The existence of upper and lower neighbours of some $\check{C}$ ech closure operators are demonstrated. The concept of simple expansions of a $\check{C}$ ech closure operator is also introduced.
\end{abstract}

AMS Subject Classification: 54A05, 03G10

Key Words: upper neighbour, topological upper neighbour, lower neighbour, simple expansion

\section{Introduction}

Edward $\check{C}$ ech in [3] introduced the concept of $\check{C}$ ech closure operators on a set $X$, as a generalisation of Kuratowski closure operators. He showed that the set of all $\check{C}$ ech closure operators on a set $X$ is a complete lattice. This lattice is complemented if and only if $X$ is finite[12]. A $\check{C}$ ech closure operator on a set $X$ is left fixed by every automorphism of $L(X)$ if and only if it is

Received: July 30, 2015

(C) 2015 Academic Publications, Ltd.

${ }^{\S}$ Correspondence author url: www.acadpubl.eu 
quasi discrete[13]. Here we consider some problems related to adjacency in the lattice of $\check{C}$ ech closure operators on a set $X$. Hewitt introduced the concept of topological expansions for strengthening a given topology [4]. If $\tau$ is topology on a given set $X$ and $A$ is a subset of $X$ not belonging to $\tau$, then the simple expansion of $\tau$ by $A$ is the smallest topology containing $\tau$ and $A$. We also investigate simple expansions in $\mathrm{L}(\mathrm{X})$.

\section{Preleminaries}

Let $X$ be a set. A Cech closure operator on a set $X$ is a function

$$
V: P(X) \rightarrow P(X)
$$

satisfying

$$
V(\phi)=\phi, \quad A \subseteq V(A),
$$

and $V(A \cup B)=V(A) \cup V(B)$ for every $A, B \in P(X)$. Here $P(X)$ denotes the power set of $X$. For brevity we call $V$ a closure operator on $X$ and the pair $(X, V)$ a closure space.

A subset $S$ of a closure space $(X, V)$ is said to be closed if $V(S)=S$, and is said to be open if its complement is closed. The collection of all open sets in a closure space $(X, V)$ is a topology on $X$, called the topology associated with $V$. A closure operator $V$ is said to be topological if and only if $V$ is idempotent.

Let $I: P(X) \rightarrow P(X)$ be given by

$$
I(A)= \begin{cases}\phi & ; \quad \text { if } A=\phi \\ X & ; \text { otherwise }\end{cases}
$$

Then $I$ is a closure operator on $X$. This closure operator is the topological closure operator associated with the indiscrete topology on $X$ and is called the indiscrete closure operator. The closure operator $D$ on $X$ given by $D(A)=A$ for all $A \in P(X)$, is the topological closure operator associated with the discrete topology on $X$, called the discrete closure operator.

Let $V_{1}, V_{2}$ be two closure operators on set $X$. Then $V_{1}$ is said to be coarser than $V_{2}$ if $V_{2}(A) \subseteq V_{1}(A)$ for every $A \in P(X)$ and is denoted by $V_{1} \leq V_{2}$. This relation in the set of all closure operators on $X$ is a partial order. The set of all closure operators on $X$ forms a lattice under this partial order and is denoted by $\mathrm{L}(\mathrm{X})$. The smallest element of this lattice is the indiscrete closure operator $I$ and the largest is the discrete closure operator $D$.

Let $\left\{V_{a} ; a \in \mathscr{A}\right\}$ where $\mathscr{A}$ is some indexing set, be a non empty collection of closure operators on $L(X)$. A closure operator $V$ which is the infimum of $\left\{V_{a}\right\}$ 
in $L(X)$ is given by $V(A)=\underset{a \in \mathscr{A}}{\cup} V_{a}(A)$ for $A \in P(X)$. If $U$ is the supremum of a non empty collection $\left\{V_{a}\right\}$ in $L(X)$ and $A \in P(X)$, then $x \in U(A)$ if and only if for each finite cover $\left\{A_{1}, A_{2}, \ldots, A_{n}\right\}$ of $A$, there exists an $A_{i}$ such that $x \in V_{a}\left(A_{i}\right)$ for each $a \in \mathscr{A}$.

For $x, y \in X, x \neq y$, define $V_{x, y}$ on $P(X)$ as follows.

$$
V_{x, y}(A)= \begin{cases}\phi & ; \text { if } A=\phi \\ X-\{y\} & ; \text { if } A=\{x\} \\ X & ; \text { otherwise. }\end{cases}
$$

Then $V_{x, y}$ is a closure operator on $X$. Such closure operators are called infra closure operators on $X$. They are precisely the atoms of $L(X)$.

Dual atoms in the lattice of topologies are called ultra topologies. Frölich proved that the ultra topologies on $X$ are precisely the topologies of the form $P(X-\{a\}) \cup \mathscr{U}$ where $a \in X$ and $\mathscr{U}$ is an ultra filter on $X$, not containing $\{a\}$. The closure operator associated with the ultra topology $P(X-\{a\}) \cup \mathscr{U}$ is given by

$$
V(A)=\left\{\begin{array}{lll}
A & ; & \text { if } a \in A \text { or } X-A \in \mathscr{U} \\
A \cup\{a\} & ; \quad \text { otherwise }
\end{array}\right.
$$

and is called the ultra closure operator. In $L(X)$, it is proved that infra closure operators are smaller than or equal to any non principal ultra closure operator[12].

The co-finite closure operator on $X$ is given by the function $C_{0}: P(X) \rightarrow$ $P(X)$ defined by

$$
C_{0}(A)=\left\{\begin{array}{lll}
A & ; & \text { if } A \text { is finite } \\
X & ; & \text { otherwise }
\end{array}\right.
$$

More generally we define $C_{\alpha}$ on $P(X)$, where $\alpha$ is any infinite cardinal number such that $\alpha \leq|X|$, by,

$$
C_{\alpha}(A)=\left\{\begin{array}{lll}
A & ; & \text { if }|A|<\alpha \\
X & ; & \text { otherwise }
\end{array}\right.
$$

When $\alpha=\aleph_{0}$, we get that $C_{\alpha}=C_{\aleph_{0}}=C_{0}$. 


\section{On Upper Neighbours of Čech Closure Operators}

In this section, we discuss some properties related to the adjacency in the lattice $L(X)$ of closure operators. Here various types of upper neighbours in $L(X)$ like topological upper neighbours, strictly topological upper neighbours are defined and investigated.

Definition 1. Let $U$ and $V$ be two closure operators on $X$ such that $U<V$. Then $V$ is called an upper neighbour of $U$ if for a closure operator $W$ on $X$ such that $U \leq W \leq V$, either $U=W$ or $W=V$.

Remark 2. If $V$ is an upper neighbour of $U$, then $U$ is called a lower neighbour of $V$. In this case, $U$ and $V$ are said to be adjacent.

\section{Example 3.}

(a) The closure operator $V_{x, y}$ and the indiscrete closure operator $I$ are adjacent. $V_{x, y}$ is an upper neighbour of $I$.

(b) The discrete closure operator $D$ and the ultra closure operator are adjacent.

Definition 4. Let $U$ and $V$ be two topological closure operators on $X$ such that $U<V$. Then $V$ is called a topological upper neighbour of $U$, if $W$ is any closure operator such that $U \leq W \leq V$, then $W$ is not topological. If $V$ is a topological upper neighbour of the topological closure operator $U$, then $U$ is a topological lower neighbour of $V$. In this case $U$ and $V$ are said to be topologically adjacent.

Definition 5. Let $U$ and $V$ be two topological closure operators on $X$. Then $V$ is called a strict topological upper neighbour of the topological closure operator $U$ if there is no closure operator $W$ on $X$ such that $U<W<V$. If $V$ is a strict topological upper neighbour of the topological closure operator $U$, then $U$ is a strict topological lower neighbour of $V$.Then $U$ and $V$ are said to be strictly topologically adjacent.

Example 6. The discrete closure operator $D$ and an ultra closure operator are strictly topologically adjacent.

Remark 7. (i) Let $V$ be a closure operator on $X$. Then for $x, y \in$ $X, x \neq y, V_{x, y} \nless \leq V$ if and only if $y \in V(\{x\})$. 
(ii) Let $V$ be a closure operator on $X$ such that $V \neq I$, and $V_{x, y} \not \leq V$, then the closure operator $U=V \vee V_{x, y}$ is given by

$$
U(S)= \begin{cases}V(S)-\{y\}, & \text { if } x \in S \text { and } y \notin V(S-\{x\}) \\ V(S) & \text { otherwise. }\end{cases}
$$

Then clearly, $U$ is finer than $V$, but $U$ need not be an upper neighbour of $V$.

Lemma 8. Let $V$ be a closure operator on $X$ such that $V \neq I$ on $L(X)$. For $x, y \in X, x \neq y$, let $V_{x, y} \not \leq V$. Then the closure operator $U=V \vee V_{x, y}$ is an upper neighbour of $V$ if and only if $y$ is in the closure of every element of $X$.

Proof. If $U$ is an upper neighbour of $V$ and $W$ is a closure operator on $X$ such that $V \leq W \leq U$, then either $V=W$ or $W=U$. Suppose $W \neq V$. Then

$$
W(S)= \begin{cases}V(S)-\{y\} & ; \text { if } S=\{x\} \\ V(S) & ; \text { otherwise. }\end{cases}
$$

is the least closure operator finer than $V$ and coarser than $U$. Therefore $W=U$ implies $y$ is in the closure of every element of $X$.

On the other hand

$$
U(S)= \begin{cases}V(S)-\{y\} & ; \text { if } S=\{x\} \\ V(S) & ; \text { otherwise }\end{cases}
$$

Obviously $U$ is an upper neighbour of $V$.

Example 9. Let $X=\{x, y, z, p\}, V$ be a closure operator on $X$ defined by $V(\phi)=\phi, V(\{x\})=\{x, y\}=V(\{y\}), V(\{z\})=\{x, y, z\}, V(\{p\})=\{x, y, p\}$. Then $U=V \vee V_{x, y}$ and $V$ are strictly topologically adjacent.

In the following results $X$ is an infinite set and $\alpha$ denotes an infinite cardinal number such that $\alpha \leq|X|$.

Definition 10. Let $a, b \in X$ with $a \neq b$. Define a function $C_{a, b}^{\alpha}: P(X) \rightarrow$ $P(X)$ by,

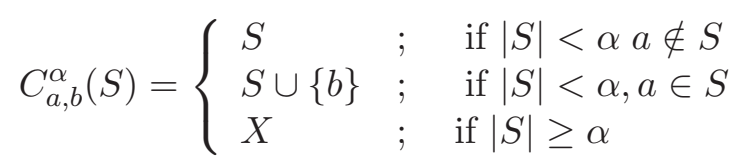

Then $C_{a, b}^{\alpha}$ is a closure operator on $X$ and $C_{a, b}^{\alpha}<C_{\alpha}$.

Theorem 11. The closure operator $C_{a, b}^{\alpha}$ is a lower neighbour $C_{\alpha}$ and any lower neighbour of $C_{\alpha}$ is of the form $C_{a, b}^{\alpha}$. 
Proof. Let $V$ be any closure operator satisfying $C_{a, b}^{\alpha} \leq V \leq C_{\alpha}$. Then for any $S$ with $|S| \geq \alpha, V(S)=X$. If $x \in X, x \neq a$, then $\{x\} \subseteq V(\{x\}) \subseteq$ $C_{a, b}^{\alpha}(\{x\})$. Thus $V(\{x\})=\{x\}$ for every $x \in X, x \neq a$. Also since $C_{a, b}^{\alpha}<V$, $\{a\} \subseteq V(\{a\}) \subset C_{a, b}^{\alpha}(\{a\})=\{a, b\}$. Hence $V(\{a\})=\{a\}$. This shows that $V=C_{\alpha}$ and thus $C_{a, b}^{\alpha}$ is a lower neighbour of $C_{\alpha}$. If $V \in L(X)$ and $V<C_{\alpha}$, then there is some $a \in X$ with $V(\{a\}) \neq\{a\}$. If $|S| \geq \alpha$, since ' $V<C_{\alpha}$, we have $C_{a, b}^{\alpha}(S)=C_{\alpha}(S)=X \subseteq V(S)$. Thus $V(S)=X$. If $S \subset X,|S|<\alpha$ and $a \notin S$, then $S=C_{a, b}^{\alpha}(S)=C_{\alpha}(S) \subseteq V(S)$. If $|S|<\alpha$ and $a \in S$, then $b \in V(S)$ and $C_{a, b}^{\alpha}(S)=S \cup\{b\}$. Hence $C_{a, b}^{\alpha}(S)=S \cup\{b\} \subseteq V(S)$. Thus $V \leq C_{a, b}^{\alpha}$.

Remark 12. If $\alpha=\aleph_{0}$, then $C_{a, b}^{\alpha}$ is given by

$$
W_{a, b}(S)= \begin{cases}S & ; \quad \text { if } \mathrm{S} \text { is finite and } a \notin S \\ S \cup\{b\} & ; \quad \text { if } \mathrm{S} \text { is finite and } a \in S \\ X & ; \quad \text { if } \mathrm{S} \text { is infinite. }\end{cases}
$$

and is a lower neighbour of $C_{0}$, the co-finite closure operator and any lower neighbour of $C_{0}$ is of the form $W_{a, b}$.

Definition 13. [13] Let $x \in X$. Suppose $A$ is an infinite subset of $X$ not containing $x$. Define $V_{A, x}: P(X) \rightarrow P(X)$ as

$$
V_{A, x}(S)= \begin{cases}S & ; \text { if } S \text { is finite } \\ X-\{x\} & ; \text { if } S \text { is infinite, } S-A \text { finite and } x \notin S \\ X & ; \text { otherwise. }\end{cases}
$$

Then $V_{A, x}$ is a closure operator on $X$ and $C_{0}<V_{A, x}$.

Remark 14. If $B \subseteq A \subseteq X$, then $V_{B, x} \leq V_{A, x}$.

We can generalise this definition as

Definition 15. Let $X$ be any set. Let $\alpha \leq|A|$ and $x$ an element of $(X-A)$. Then define $C_{A, x}^{\alpha}: P(X) \longrightarrow P(X)$ by,

$$
C_{A, x}^{\alpha}(S)= \begin{cases}S & \text { if }|S|<\alpha \\ X-\{x\} & \text { if }|S| \geq \alpha,|S-A|<\alpha \text { and } x \notin S \\ X & \text { otherwise }\end{cases}
$$

Then $C_{A, x}^{\alpha}$ is an element of $L(X)$ and $C_{\alpha}<C_{A, x}^{\alpha}$ and thus $C_{A, x}^{\alpha} \in\left(C_{\alpha}, D\right)$.

Remark 16. If $B \subseteq A \subseteq X$ such that $\alpha \leq|B|$, then $y \in C_{A, x}^{\alpha}(S), S \subseteq X$ implies that $y \in C_{B, x}^{\alpha}(S)$. Thus $C_{B, x}^{\alpha} \leq C_{A, x}^{\alpha}$. 
Theorem 17. If $A$ and $B$ are two distinct subsets of $X$ such that $\alpha \leq|A|$ and $\alpha \leq|B|$ and $x \in X-(A \cup B)$, then $C_{A, x}^{\alpha}$ and $C_{B, x}^{\alpha}$ are equal if and only if $|A \Delta B|<\alpha$.

Proof. Suppose $|A \Delta B|<\alpha$. Then $|A-B|<\alpha$ and $|B-A|<\alpha$. Thus we have $|S-A|<\alpha$ if and only if $|S-B|<\alpha$. Thus $C_{A, x}^{\alpha}$ and $C_{B, x}^{\alpha}$ are equal, by definition.

Conversely assume that $C_{A, x}^{\alpha}=C_{B, x}^{\alpha}$. Then $C_{A, x}^{\alpha}(A)=C_{B, x}^{\alpha}(A)=X-\{x\}$. Hence $|A-B|<\alpha$. Also $C_{A, x}^{\alpha}(B)=C_{B, x}^{\alpha}(B)=X-\{x\}$. Then $|B-A|<\alpha$. Thus $|A \Delta B|<\alpha$.

Lemma 18. The closure operator $C_{A, x}^{\alpha}$ is topological if and only if $|X-A|<\alpha$.

Proof. If $|X-A|<\alpha, C_{A, x}^{\alpha}=C_{X-\{x\}, x}^{\alpha}$ by Theorem 17 . Hence if $|S| \geq \alpha$, $|S-A|<\alpha$ and $x \notin S$, then $C_{A, x}^{\alpha}\left(C_{A, x}^{\alpha}(S)\right)=C_{A, x}^{\alpha}(X-\{x\})=X-\{x\}=$ $C_{A, x}^{\alpha}(S)$. If $|X-A| \geq \alpha$, then $C_{A, x}^{\alpha}(A)=X-\{x\}$ and $C_{A, x}^{\alpha}\left(C_{A, x}^{\alpha}(A)\right)=X$. Thus $C_{A, x}^{\alpha}$ is not topological.

Lemma 19. If $A \subset X,|A| \geq \alpha$, and $x \in X-A$, then there is a subset $D$ of $A$ such that $C_{D, x}^{\alpha}<C_{A, x}^{\alpha}$.

Proof. Since $|A| \geq \alpha$, there exists a subset $B$ of $A$ with $|B|=\alpha$. Since $\alpha+\alpha=\alpha, B$ has a subset $D$ with $|D|=\alpha$ and $|B \Delta D|=\alpha$. Hence by Theorem $17, C_{D, x}^{\alpha}<C_{B, x}^{\alpha} \leq C_{A, x}^{\alpha}$.

Lemma 20. Let $V$ be a closure operator on $X$ such that $C_{\alpha}<V$. Then $C_{A, x}^{\alpha} \leq V$ if and only if $x \notin V(A)$.

Proof. Suppose $C_{A, x}^{\alpha} \leq V$. Then $V(A) \subseteq C_{A, x}^{\alpha}(A)=X-\{x\}$. Now if $x \notin V(A), V(A) \subseteq X-\{x\}=C_{A, x}^{\alpha}(A)$. Hence the result.

Remark 21. When $\alpha=\aleph_{0}$, we get that $C_{A, x}^{\alpha}=V_{A, x}$. Then $V_{A, x} \leq V$ if and only if $x \notin V(A)$.

Theorem 22. The closure operator $C_{\alpha}$ has no upper neighbour in the lattice of closure operators.

Proof. Let $V$ be any closure operator in $L(X)$ satisfying $C_{\alpha}<V$. When $|S|<\alpha, C_{\alpha}(S)=S$. Choose $A \subset X$ with $|A| \geq \alpha$ and $V(A) \subset C_{\alpha}(A)=X$. Thus there is $x \in X-V(A)$. Hence $C_{A, x}^{\alpha} \leq V$ by Lemma 20. Thus there 
are closure operators $C_{D, x}^{\alpha}$ and $C_{B, x}$ such that $C_{D, x}^{\alpha}<C_{B, x}^{\alpha} \leq C_{A, x}^{\alpha}$. Hence $C_{\alpha}<C_{D, x}^{\alpha}<C_{B, x}^{\alpha} \leq C_{A, x}^{\alpha} \leq V$.

Remark 23. If $\alpha=\aleph_{0}, C_{\alpha}$ is the co-finite closure operator and thus above theorem says co-finite closure operator has no upper neighbour in the lattice of closure operators.

Theorem 24. Let $V$ be any closure operator on $X$ such that $C_{0}<V$. Then $V$ is the join of all closure operators of the form $V_{A, x}$ where $A$ is an infinite subset of $X$ and $x \in X-A$.

Proof. Let $U$ be the join of all closure operators of the form $V_{A, x} \leq V$. Clearly, $U \leq V$. If $U \neq V$, then there is a $B \subseteq X$ and $x \in U(B)$, but $x \notin V(B)$. If $B$ is finite, $V(B)=U(B)=B$. Thus if $B$ is infinite and $x \notin B$. Then $V_{B, x} \leq V$. Consequently, we have that $V_{B, x} \leq U$. Then $x \notin U(B)$, which is a contradiction. Hence the result.

We have by Lemma $18, V_{A, x}$ is topological if and only if $X-A$ is finite. We denote $V_{A, x}$ by $V_{x}$ when $X-A$ is finite.

Theorem 25. Let $x \in X$. Then $V_{x}$ has no upper neighbour in $\left[C_{0}, D\right]$.

Proof. Let $V \in\left[C_{0}, D\right]$ such that $V_{x}<V$. Then there is $A \subset X$ such that $V(A) \subset V_{x}(A)$. Then there is a $y \in V_{x}(A)$ such that $y \notin V(A)$. Then $y \neq x$. For, if $y=x$, then $x \notin A$. Then $x=y \in V_{x}(A)=X-\{x\}$, a contradiction. Further we claim that $A$ is infinite. For, if $A$ is finite, $y \notin V_{x}(A)=A$, a contradiction. Then $V_{A, y} \leq V$, since $y \notin V(A)$. There is an infinite subset $C$ of $A$ such that $V_{C, y}<V_{A, y}$. Let $V^{\prime}=V_{x} \bigvee V_{C, y}$. Then $V_{x}<V$ and $V_{C, y}<V_{A, y} \leq$ $V$ implies that $V^{\prime}=V_{x} \bigvee V_{C, y}<V$. Also $V_{x}<V_{x} \bigvee V_{C, y}=V^{\prime}$. Thus $V_{x}$ does not have an upper neighbour.

By modifying this proof we can also prove that $V_{A, x}$ cannot have an upper neighbour in $L(X)$.

Theorem 26. Let $A$ be an infinite subset of $X$ and $x \in X-A$. Then $V_{A, x}$ has no upper neighbour in $L(X)$.

Proof. Let $V$ be a closure operator such that $V_{A, x}<V$. Then there exists $B \subset X$ such that $V(B) \subset V_{A, x}(B)$. Then there exists $y \in V_{A, x}(B)$ such that $y \notin V(B)$. Then $B$ is infinite and $y \notin B$.

Case $(i): y \neq x$

Since $y \notin V(B)$ we get that $V_{B, y} \leq V$. By Lemma 19, there exists a subset $C$ 
of $B$ such that $V_{C, y}<V_{B, y} \leq V$. Let $V^{\prime}=V_{A, x} \bigvee V_{C, y}$. Then $V_{A, x}<V^{\prime}$ for otherwise $V_{C, y} \leq V_{A, x}$. Also we have that

$V^{\prime}<V$ since $V^{\prime}=V_{A, x} \bigvee V_{C, y}<V_{A, x} \bigvee V_{B, y} \leq V$.

Thus $V_{A, x}<V^{\prime}<V$.

Case $(i i): y=x$

Then $x \in V_{A, x}(B)$. Then $B-A$ is infinite. Let $\left\{b_{1}, b_{2}, \ldots\right\}$ be a countable infinite subset of $B-A$. Let $C=\left\{b_{2}, b_{4}, \ldots\right\}$. Then $V_{C, x}<V_{B, x}$. But $x=y \notin V(B)$.

Then $V_{B, x} \leq V$. Thus $V_{C, x}<V_{B, x}<V$. Let $V^{\prime}=V_{A \cup C, x}$.

Then $V_{A, x}<V^{\prime}$. Also $V^{\prime}=V_{A \cup C, x}<V_{A \cup B, x}, x \notin V(B)$. Also $V(A) \subset$ $V_{A, x}(A)=X-\{x\}$. Thus $x \notin V(A)$ Then $x \notin V(A \cup B)=V(A) \cup V(B)$. Thus $V_{A \cup B, x} \leq V$. Consequently, we obtain that $V_{A, x}$ has no upper neighbour in both the cases.

Remark 27. In the lattice of $T_{1}$ topologies, the smallest element co-finite topology and the atoms have upper neighbours. But in $L(X)$, co-finite closure operator $C_{0}$ and its strictly topological upper neighbour $V_{x}$ do not have an upper neighbour.

Theorem 28. Suppose $A$ and $B$ are two infinite subsets of $X, x \notin A \cup B$, then $V_{A, x} \bigvee V_{B, x}=V_{A \cup B, x}$.

Proof. By Remark 14, $V_{A, x} \leq V_{A \cup B, x}(S)$ and $V_{B, x} \leq V_{A \cup B, x}(S)$. Hence $V_{A, x} \bigvee V_{B, x} \leq V_{A \cup B, x}$.

To prove $V_{A \cup B, x} \leq V_{A, x} \bigvee V_{B, x}$, it is enough to prove that $x \notin\left(V_{A, x} \bigvee V_{B, x}\right)(A \cup B)$, by Remark 21. We have $\left(V_{A, x} \bigvee V_{B, x}\right)(A) \subseteq V_{A, x}(A)$ and $\left(V_{A, x} \bigvee V_{B, x}\right)(B) \subseteq V_{B, x}(B)$. Since $x \notin V_{A, x}(A), x \notin\left(V_{A, x} \bigvee V_{B, x}\right)(A)$. Similarly we can prove that $x \notin\left(V_{A, x} \bigvee V_{B, x}\right)(B)$. Therefore $x \notin\left(V_{A, x} \bigvee V_{B, x}\right)(A \cup$ $B)=V$.

Theorem 29. If $A_{1}, A_{2}, \ldots, A_{n}$ is a finite collection of infinite subsets of $X$ such that $x \notin A_{i}$ for any $i \in\{1,2, \ldots, n\}$, then $V_{A_{1}, x} \bigvee V_{A_{2}, x} \bigvee \ldots \bigvee V_{A_{\mathrm{n}}, x}=$ $V_{A_{1} \cup A_{2} \cup \ldots \cup A_{\mathrm{n}}, x}$.

Proof. Similar to the proof of Theorem 28. 


\section{Simple Expansions in $L(X)$}

The concept of simple expansions is very important in the study of upper neighbours in the lattice of topologies. The simple expansion $\tau(A)$ of the topology $\tau$ by a subset $A$ of $X, A \notin \tau$, is the join of $\tau$ and the atom $\{\phi, A, X\}$. In this section we introduce an analogous concept in the lattice of Cech closure operators.

Definition 30. Let $A$ be a non empty proper subset of $X$ and $x \in A$. Define, $V_{(A, x)}: P(X) \rightarrow P(X)$ by,

$$
V_{(A, x)}(S)= \begin{cases}\phi & ; \text { if } S=\phi \\ X-\{x\} & ; \text { if } S \subseteq X-A \text { and } S \neq \phi \\ X & ; \quad \text { otherwise }\end{cases}
$$

Then $V_{(A, x)}$ is a closure operator on $X$.

Remark 31. 1. If $A=X-\{y\}, x, y \in X$ and $x \neq y$, then $V_{(A, x)}$ is

$$
V_{(X-\{y\}, x)}(S)= \begin{cases}\phi & ; \quad \text { if } S=\phi \\ X-\{x\} & ; \quad \text { if } S=\{y\} \\ X & ; \quad \text { otherwise }\end{cases}
$$

This shows that $V_{(X-\{y\}, x)}=V_{y, x}$.

2. If $A=\{x\}$

$$
V_{(\{x\}, x)}(S)= \begin{cases}\phi & ; \quad \text { if } S=\phi \\ X-\{x\} & ; \quad \text { if } x \notin S \neq \phi \\ X & ; \quad \text { otherwise }\end{cases}
$$

Theorem 32. The closure operator $V_{(A, x)}$ is topological if and only if $A=\{x\}$.

Proof. If $S=\phi$ or $S=X, V_{(\{x\}, x)}\left(V_{(\{x\}, x)}(S)\right)=V_{(\{x\}, x)}(S)$. If $x \notin S$, $V_{(\{x\}, x)}\left(V_{(\{x\}, x)}(S)\right)=V_{(\{x\}, x)}(X-\{x\})=X-\{x\}=V_{(\{x\}, x)}(S)$. Conversely assume that $V_{(A, x)}$ is topological. Let $S \subseteq X-A, S \neq \phi$. Then

$V_{(A, x)}(X-\{x\})=X-\{x\}$. Thus $X-\{x\} \subseteq X-A$. Since $A$ is non empty $A=\{x\}$.

Remark 33. The closed subsets of $X$ with respect to the closure operator $V_{(\{x\}, x)}$ are $\phi, X-\{x\}$ and $X$. Hence $V_{(\{x\}, x)}$ is the topological closure operator of the infra topology $\{\phi,\{x\}, X\}$. The closure operator $V_{(X-\{y\}, x)}$ is not topological when $|X| \geq 3$. 
Lemma 34. If $A$ is a proper subset of $X$, then for every closure operator of the form $V_{(A, x)}$ on $X$, there is an element $y \in X-A$ such that, $V_{(X-\{y\}, x)} \leq$ $V_{(A, x)} \leq V_{(\{x\}, x)}$.

Proof. If $|X|=2$, then $A \neq X$, otherwise $V_{(A, x)}=I$, the indiscrete closure operator. In this case, $V_{(X-\{y\}, x)}=V_{(A, x)}=V_{(\{x\}, x)}$. Let $|X| \geq 3$. Then $A \neq X$. Let $y \in X-A$, then clearly, $V_{(X-\{y\}, x)} \leq V_{(A, x)} \leq V_{(\{x\}, x)}$.

If $|X| \geq 3$, then the closure operator $V_{(A, x)}$ is not topological for any proper subsets $A$ of $X$ such that $A$ is different from $\{x\}$. In this case the topology associated with $V_{(A, x)}$ is the indiscrete topology.

Using the above concept we introduce simple expansion of a closure operator in the following way.

Definition 35. Let $V$ be any closure operator on $X$ and $A$ be a subset of $X$ such that $x \in A$. The closure operator $V_{A}^{x}=V \bigvee V_{(A, x)}$ is called a simple expansion of $V$ by $A$ at $x$. The closure operator $V_{A}^{x}$ is given by,

$$
V_{A}^{x}(S)= \begin{cases}V(S)-\{x\} & ; \quad \text { if } S \cap(X-A) \neq \phi \text { and } x \notin V(S \cap A) \\ V(S) & ; \text { otherwise }\end{cases}
$$

Lemma 36. $V_{A}^{x}=V$ if and only if $x \notin V(X-A)$.

Proof. Suppose $V_{A}^{x}(S)=V$ then we have $V_{A}^{x}(S)=V(S)$ for every subset $S$ of $X$. Hence if $S \cap(X-A) \neq \phi$ and $x \notin V(S \cap A)$ then $x \notin V(S)$. This implies $x \notin V(X-A)$. If $x \notin V(X-A)$, then from the definition $V_{A}^{x}=V$.

Theorem 37. The simple expansion $C_{0}^{x}$ of $C_{0}$ by $A$ at $x$ is topological if and only if $A$ is finite.

Proof. We have,

$$
C_{0 A}^{x}(S)= \begin{cases}S & ; \quad \text { if } S \text { is finite } \\ X-\{x\} & ; \quad \text { if } S \cap(X-A) \neq \phi \text { and } x \notin C_{0}(S \cap A) \\ X & ; \quad \text { otherwise }\end{cases}
$$

If $C_{0 A}^{x}$ is topological, then $C_{0} x\left(C_{0 A}^{x}(S)\right)=C_{0 A}^{x}(S)$ for every subset $S$ of $X$. Thus if $S \cap(X-A) \neq \phi$ and $x \notin C_{0}(S \cap A)$, then $C_{0}{ }_{A}^{x}(S)=X-\{x\}$ and $C_{0}{ }_{A}^{x}(X-\{x\})=X-\{x\}$. This is when $x \notin C_{0}(S \cap A)$ implies $S \cap A$ is finite. Since $C_{0} x(X-\{x\})=X-\{x\}, A$ must be finite. If $A$ is finite, then for every infinite set $S$ not containing $x$, we have $x \notin C_{0}(S \cap A)$. Therefore for every infinite subset $S$ of $X$ such that $x \notin S, C_{0}^{x}(S)=X-\{x\}$ and $C_{0 A}^{x}(X-\{x\})=X-\{x\}$. 
Lemma 38. The closure operator $V_{A, x}$ is a simple expansion of $C_{0}$ by $X-A$ at $x$. In other words $V_{A, x}=V_{(X-A, x)} \bigvee C_{0}$.

Proof. As we have noted before, $C_{0} \leq V_{A, x}$ and $V_{(X-A, x)} \leq V_{A, x}$. Therefore $V_{(X-A, x)} \bigvee C_{0} \leq V_{A, x}$. Let $S \subseteq X$. Now suppose $x \in\left(V_{(X-A, x)} \bigvee C_{0}\right)(S)$ Then for every finite cover $\left\{S_{i}\right\}$ of $S$, there exists a $S_{j}$ such that $x \in C_{0}\left(S_{j}\right)$ and $x \in V_{(X-A, x)}\left(S_{j}\right)$. Thus $S_{j}$ is infinite and $S_{j}$ not contained in $A$. Since $S_{j} \subseteq S$, we have $x \in V_{A, x}(S)$. Hence $V_{A, x} \leq V_{(X-A, x)} \bigvee C_{0}$.

Remark 39. 1. The simple expansion $C_{0}^{x}$ of $C_{0}$ by $A$ at $x$ when $A$ is finite is $V_{x}$.

2. The simple expansion $C_{0}{ }_{A}^{x}$ of $C_{0}$ by $A$ at $x$ and the simple expansion $C_{0} \stackrel{x}{B}$ of $C_{0}$ by $B$ at $x$ are equal if and only if $A \Delta B$ is finite.

3.Let $V$ be a closure operator on $X$. If $X-A$ is a singleton set, say $\{y\}$, then the simple expansion $V_{A}^{x}$ of $V$ by $A$ at $x$ is an upper neighbour of $V$ if and only if $x$ is in the closure of every element of $X$. (Refer Lemma 8)

Proposition 40. Let $V$ be a closure operator on $X$, $B \subseteq A \subseteq X$ and $x \in B$. Then $V_{A}^{x} \leq V_{B}^{x}$.

Proof. If $B \subseteq A$, then $V_{(A, x)} \leq V_{(B, x)}$, Thus $V \bigvee V_{(A, x)} \leq V \bigvee V_{(B, x)}$. Hence $V_{A}^{x} \leq V_{B}^{x}$

Example 41. The converse of this result is not true. Let $X$ be the set of all positive integers. Take $A=\{x \in X \mid x=1$ or $x$ is even $\}$ and $B=\{x \in X \mid x=3$ or $x$ is even $\}$. Then $X-A, X-B$ are not comparable and $A \Delta B$ is finite. But $C_{0(X-A)}^{x}$ and $C_{0(X-B)}^{x}$ are equal.

\section{Acknowledgments}

The second author acknowledges the financial support from U.G.C., Govt. of India.

\section{References}

[1] Agashe P. and Levine N., Adjacent Topologies, J. Math. Tokushima Univ., 7 (1973), 21-35.

[2] Birkhoff G., Lattice Theory, Amer. Math. Soc. Colloq. Pub. 25, Third Edn. (1967), doi: http//dx.doi.org/10.2307/2268183. 
[3] C̆ech E., Topological Spaces, Rev. Ed. Wiley, New York(1966), Library of Congress Catalogue Card Number 66-13066.

[4] Hewitt E., A Problem of Set Theoretic Topology, Duke Math. J., 10 (1943), 309-333, doi: 10.1215/S0012-7094-43-01029-4.

[5] Kunheenkutty M., On Simple Expansions of Topologies, Proceedings, K. M. A. National Seminar on New Vistas in Topology, KKTM Govt College, Pullut, Kodungallur, Kerala (2005), 11-15.

[6] Kunheenkutty M., Comparison of Simple Expansions of Topologies, Proceedings, International Seminar on Recent Trends in Topology and its Applications, St. Joseph's College, Irinjalakuda, Kerala (2009), 99-105.

[7] Kunheenkutty M., Some Problems on Local Properties of the Lattice of Topologies, Thesis for Ph. D. Degree, University of Calicut(2013).

[8] Kunheenkutty M., On Simple Expansions of Topologies by Singletons, Bulletin of Kerala Mathematics Association, 10, No. 1 (2013), 1-6.

[9] Larson R. E. and Andima S.J., The Lattice of Topologies: A Survey, Rocky Mountain J. Math., 5 (1975), 177-198, doi: 10.1216/RMJ-1975-5-2-177.

[10] Levine N., Simple Extensions of Topologies, Amer. Math. Mon., 71 (1964) 22-25, doi: $10.2307 / 2311297$.

[11] Ramachandran P.T., Some Problems in Set Topology Relating Group of Homeomorphisms and Order, Thesis for Ph. D. Degree, CUSAT (1985).

[12] Ramachandran P.T., Complementation in the Lattice of Cech Closure Operators, Indian J. Pure. App. Math., 18 No. 2 (1987), 152-158.

[13] Ramachandran P.T., Fixed Points of the Lattice of Cech Closure Operators, Proceedings, U.G.C. Sponsored National Seminar on Discrete Analysis at Sri Ramakrishna Mission Vidyalaya College of Arts and Science (Autonomous), Coimbatore 641020 (1999), 29-31.

[14] Ramachandran P.T., Complemented Elements in the Lattice of Čch Closure Operators, J. Comp. \& Math. Sci. 3 No. 5 (2012), 498-501.

[15] Robert R. Stoll, Set Theory and Logic, Dover Publication, Inc. New York(1961), Library of Congress Catalog Card Number 79-87812. 
[16] Steiner A. K., The Lattice of Topologies: Stucture and Complementation, Trans. Amer. Math. Soc. 122 (1966) 379-398, doi: 10.2307/1994555. 